\title{
RECIDIVAS LOCAIS APÓS TRATAMENTO CIRÚRGICO DO CARCINOMA EPIDERMÓIDE DE CABEÇA E PESCOÇO EM ESTÁGIOAVANÇADO
}

\author{
LOCAL RECURRENCE AFTER SURGICAL TREATMENT FOR ADVANCED SQUAMOUS \\ CELL CARCINOMA OF THE HEAD AND NECK
}

\author{
Ali Amar'; Otávio Alberto Curioni, TCBC-SP'; \\ Sergio Altino Franzi ${ }^{1}$; Abrão Rapoport, ECBC-SP
}

\begin{abstract}
RESUMO: Objetivo: Avaliar os resultados do tratamento de resgate das recidivas locais nos pacientes com carcinoma epidermóide das vias aerodigestivas superiores submetidos a tratamento cirúrgico. Método: Foram revisados os prontuários de 943 pacientes submetidos à ressecção do tumor primário e esvaziamento cervical entre 1977 e 1996, e selecionados 107 pacientes com recidiva no sítio primário. Foram avaliados os resultados do tratamento de resgate, bem como os motivos para a não realização do mesmo. Resultados: A principal queixa no diagnóstico da recidiva foi dor. O tratamento de resgate foi realizado em $45 \%$ dos pacientes e o principal motivo para a não realização do mesmo foi a extensão da recidiva. Foram resgatados $54 \%$ dos pacientes com recidiva no estádio rT1T2 em comparação com $31 \%$ dos pacientes rT3T4 (p=0,04). Apenas quatro(8\%) dos pacientes resgatados estavam assintomáticos por período superior a 12 meses após o re-tratamento, todos resgatados com cirurgia. $\mathrm{O}$ intervalo de tempo entre o tratamento inicial e a recidiva apresentou mediana de oito meses e as novas recidivas ocorreram na mediana de cinco meses. Conclusão: As recidivas locais após tratamento cirúrgico extenso apresentam prognóstico reservado (Rev. Col. Bras. Cir. 2005; 32(2): 60-63).
\end{abstract}

Descritores: Recidiva local de neoplasia; Neoplasias de cabeça e pescoço; Carcinoma de células escamosas.

\section{INTRODUÇÃO}

As recidivas locais são a causa mais comum de falha do tratamento dos pacientes com carcinoma epidermóide das vias aerodigestivas superiores em estádio avançado ${ }^{1-3}$. A recidiva, ou mais propriamente a persistência da doença, pode representar uma falha técnica relacionada ao planejamento terapêutico ou um fenótipo tumoral mais agressivo. O seguimento a curtos intervalos tem por objetivo fazer o diagnóstico precoce das recidivas, que habitualmente ocorrem nos primeiros dois anos após o tratamento inicial, tendo em vista o resgate ${ }^{1,2,4}$. Muitos destes pacientes passam por ressecções extensas e radioterapia pós-operatória, o que dificulta o retratamento com intento curativo e explica o mau prognóstico desta condição. O presente estudo avalia a manifestação clínica e os resultados do tratamento das recidivas locais em pacientes submetidos a grandes ressecções por carcinoma epidermóide de cabeça e pescoço.

\section{MÉTODO}

Foram revisados os prontuários de 943 pacientes submetidos à ressecção do tumor primário e esvaziamento cervical entre 1977 e 1996, no Departamento de Cirurgia de Cabeça e Pescoço e Otorrinolaringologia do Hospital Heliópolis, com carcinoma epidermóide da boca, orofaringe, hipofaringe e laringe. Destes, 154 pacientes apresentaram recidiva no sítio primário durante o seguimento. Foram excluídos os pacientes submetidos a tratamento prévio e aqueles com recidivas após três anos ou mais do tratamento inicial, bem como aqueles nos quais a suspeita clínica de recidiva no sítio primário não foi confirmada pela biópsia ou evolução clínica, restando 107 pacientes. Quanto ao gênero, a casuística é composta por 99 homens e oito mulheres. Em relação ao sítio primário, 61 pacientes apresentavam tumor inicial na boca, 15 na orofaringe, 17 na hipofaringe e 14 na laringe. Quanto ao estadiamento, nove pacientes apresentavam doença no estádio II, 42 no estádio III e 56 no estádio IV. As recidivas locais ocorreram isoladamente em 78 pacientes. Em 27 casos as recidivas foram loco-regionais e em dois pacientes a recidiva local estava associada à metástase à distância. Todos os pacientes com tumor primário na laringe foram inicialmente submetidos à laringectomia total. A recidiva local nestes casos ocorreu adjacente à linha de sutura na faringe, e as peritraqueostoma não foram consideradas. Foram revisadas as queixas presentes no diagnóstico da recidiva, bem como o estadiamento TNM da UICC-AJCC do tumor inicial e da recidiva, conforme a revisão de 2002. Foram avaliados os resultados do retratamento (resgate) ou os motivos para a não realização do mesmo. Entre os pacientes submetidos ao tratamento de resgate, foi considerado sucesso terapêutico um período livre de doença igual ou superior a 12 meses. As diferenças entre os grupos foram avaliadas pelo teste exato de Fisher, sendo consideradas significativas quando $\mathrm{p}<0,05$.

\section{RESULTADOS}

A principal queixa no diagnóstico da recidiva foi dor, seguida pela queixa de ferida ou "caroço", conforme mostra a Tabela 1 . O tratamento de resgate foi realizado em 49 (45\%)

1. Cirurgião do Departamento de Cirurgia de Cabeça e Pescoço e Otorrinolaringologia do Hospital Heliópolis, Hosphel. São Paulo, Brasil.

Trabalho realizado no Departamento de Cirurgia de Cabeça e Pescoço e Otorrinolaringologia do Hospital Heliópolis, Hosphel. São Paulo, Brasil. 
Tabela 1 - Queixa principal no diagnóstico da recidiva.

\begin{tabular}{lr}
\hline Queixa & n \\
\hline Dor & 40 \\
Ferida/Caroço & 25 \\
Disfagia & 4 \\
Outras queixas & 19 \\
Não relatado & 19 \\
\hline
\end{tabular}

Tabela 2 - Motivo da não realização de resgate cirúrgico.

\begin{tabular}{lr}
\hline Motivo & n \\
\hline Extensão da recidiva & 51 \\
Condições clínicas & 7 \\
Recusa/Sem retorno & 7 \\
Óbito pré-tratamento & 2 \\
Sem registro & 15 \\
\hline
\end{tabular}

pacientes. O resgate com cirurgia, associada ou não à radioterapia, foi realizado em 25 casos. Houve um óbito no pós-operatório imediato e em três casos a ressecção foi incompleta. A radioterapia foi empregada isoladamente ou associada à quimioterapia em 24 casos. A principal causa para a não realização de resgate cirúrgico foi a extensão da recidiva, conforme mostra a Tabela 2. Cinco pacientes foram tratados de forma paliativa com quimioterapia.

Os pacientes previamente irradiados (radioterapia pós-operatória) foram resgatados em $34 \%$ dos casos, em comparação a $60 \%$ dos pacientes não irradiados $(\mathrm{p}=0,01)$, embora a proporção de resgates cirúrgicos tenha sido similar (23\% e $22 \%$ respectivamente). Foram resgatadas $54 \%$ das recidivas rT1 e rT2 e $31 \%$ das recidivas rT3 e rT4 $(\mathrm{p}=0,04)$. A Tabela 3 apresenta a relação entre o estadiamento da lesão inicial $(\mathrm{T})$ e da recidiva $(\mathrm{rT})$ com a possibilidade de resgate. Dos 107 pacientes, 10 apresentavam recidivas de estádio rTx e em 16 casos não haviam informações referentes ao estadiamento nos prontuários. Apenas quatro (8\%) pacientes resgatados estavam vivos e assintomáticos 12 meses após o resgate, sendo que todos foram resgatados com cirurgia. Houve um óbito não relacionado ao câncer três meses após o resgate.

O intervalo de tempo entre o tratamento inicial e a primeira recidiva local apresentou média de 10 meses e mediana de oito meses. Dos 49 pacientes resgatados, 17 persistiram com doença após o retratamento. Entre os pacientes que apresentaram uma nova recidiva, a média e a mediana do intervalo entre o resgate e a nova recidiva foram respectivamente sete meses e cinco meses.

Entre os 24 pacientes resgatados com radioterapia, quatro foram perdidos de seguimento e $12(60 \%)$ dos 20 pacientes remanescentes persistiam com doença ao término do tratamento. Todos os oito pacientes com doença controlada desenvolveram nova recidiva em um intervalo de um a oito meses.

Não foi observada relação entre o estadiamento do tumor inicial ou da recidiva com o intervalo livre de doença após o tratamento inicial, demonstrado na Tabela 4.

\section{DISCUSSÃO}

As recidivas locais ocorrem com maior freqüência nos primeiros dois anos pós-tratamento, sendo que a partir do terceiro ano a incidência de segundo tumor primário supera a taxa de recidiva local ${ }^{1,2}$. Considerando que um segundo tumor pode se desenvolver na área da ressecção prévia, o presente estudo considerou como recidivas locais apenas as lesões diagnosticadas nos primeiros três anos. A definição é arbitrária, porque é impossível distinguir clinicamente se uma lesão que se desenvolve na área da ressecção prévia constitui doença residual ou uma nova neoplasia dentro do mesmo campo de cancerização ${ }^{5}$. Esta distinção tem grande influência sobre os resultados do tratamento, pois o resgate de um segundo tumor encerra um prognóstico melhor. Adicionalmente, os melhores resultados no tratamento de resgate são observados entre os pacientes com tumor da laringe ou naqueles inicialmente não submetidos a tratamento cirúrgico. $\mathrm{O}$ resgate com laringectomia total em um paciente inicialmente submetido à laringectomia parcial ou radioterapia apresenta grande probabilidade de controle local, o que favoreceu o desenvolvimento de estratégias de preservação de órgão neste sítio anatômico ${ }^{1,6,7}$. A presente casuística, no entanto, apresenta um outro perfil de pacientes, já submetidos inicialmente à laringectomia total. Nesta situação as recidivas dificilmente são passíveis de resgate, o que explica a má evolução destes pacientes. Assim como o estadiamento da recidiva, o estadiamento inicial também pode influenciar a indicação do resgate e o prognóstico individual ${ }^{3,6}$. O estadiamento inicial está relacionado ao tamanho da ressecção prévia e, consequentemente, estaria relacionado à maior ou menor possibilidade de resgate cirúrgico. Mesmo uma recidiva pequena pode ser irressecável se a cirurgia prévia alcançou os limites anatômicos de ressecabilidade. Contudo, uma recidiva de pequenas dimensões sempre apresenta maior possibilidade de

Tabela 3 - Estadiamento inicial e da recidiva em relação ao resgate e a sobrevida livre de doença*.

\begin{tabular}{lcccc}
\hline & \multicolumn{2}{c}{ Resgate } & \multicolumn{2}{c}{ SLD > 12 meses } \\
& Sim & Não & Sim & Não \\
\hline T1-2 & $13(45 \%)$ & $16(55 \%)$ & 2 & 11 \\
T3-4 & $34(44 \%)$ & $44(56 \%)$ & 2 & 32 \\
rT1-2 & $18(54 \%)$ & $15(46 \%)$ & 2 & 16 \\
rT3-4 & $15(31 \%)$ & $33(69 \%)$ & 2 & 13 \\
\hline
\end{tabular}

* estadiamento não disponível para todos os pacientes.

Tabela 4 - Estadiamento do tumor primário e da recidiva em relação ao intervalo de tempo entre o tratamento inicial e a recidiva*.

\begin{tabular}{|c|c|c|}
\hline & \multicolumn{2}{|c|}{ Tempo } \\
\hline & $\leq 6$ meses & $>6$ meses \\
\hline T1-2 & $13(45 \%)$ & $16(55 \%)$ \\
\hline $\mathrm{T} 3-4$ & $30(38 \%)$ & $48(62 \%)$ \\
\hline rT1-2 & $12(36 \%)$ & $21(64 \%)$ \\
\hline rT3-4 & $17(35 \%)$ & $31(65 \%)$ \\
\hline
\end{tabular}

* estadiamento não disponível para todos os pacientes. 
resgate do que uma recidiva extensa. O sítio primário e o tratamento prévio também podem influenciar a indicação do resgate, fazendo com que o estadiamento da recidiva não tenha o mesmo significado prognóstico que o estadiamento representa no tratamento inicial ${ }^{7,8}$.

Entre os pacientes resgatados com radioterapia, nenhum apresentou controle da doença por mais de oito meses. A re-irradiação é um assunto controverso devido ao grande risco de complicações graves, especialmente quando há sobreposição dos campos irradiados e doses cumulativas superiores a 125 Gy ${ }^{9,10,11}$. A re-irradiação, contudo, é pouco efetiva nos pacientes previamente submetidos à cirurgia, com resposta completa em $48 \%$ e $14 \%$ dos pacientes com ou sem cirurgia prévia, respectivamente ${ }^{9}$. É relatado melhor prognóstico no resgate das recidivas tardias, possivelmente relacionado à maior incidência de segundo tumor ${ }^{9,10}$.

O papel da quimioterapia no tratamento de resgate não está definido. A resposta ao tratamento é observada em aproximadamente $25 \%$ a $40 \%$ dos pacientes, embora as respostas completas sejam observadas em apenas $10 \%$ dos ca$\operatorname{sos}^{12-14}$. A quimioterapia parece não alterar significativamente a sobrevida global, mas os pacientes que tem resposta completa apresentam maior sobrevida em comparação aos pacientes com resposta parcial ou sem resposta. Como a quimioterapia nestes pacientes é um tratamento paliativo, não se justificam esquemas terapêuticos com elevada toxicidade.
A dor é o sintoma mais freqüente e pode anteceder o diagnóstico histológico da recidiva em dois a quatro meses ${ }^{15}$. O diagnóstico precoce é importante não apenas pela maior probabilidade de resgate, mas também porque possibilita um tratamento mais simples ${ }^{7}$. A proposta de uma cirurgia extensa, com elevada morbi-mortalidade, em um paciente debilitado e com grande risco de complicações, deve ser questionada diante da pequena probabilidade de controle da doença. No tratamento de resgate, os aspectos relacionados à qualidade de vida adquirem maior importância. O efeito do tratamento de resgate sobre a sobrevida global é difícil de ser avaliado, uma vez que os pacientes não resgatados apresentam doença mais avançada e pior estado geral, características que se relacionam com pior prognóstico. Apesar da persistência da doença, os pacientes com recidiva local podem apresentar longa sobrevida, semelhante ao observado na evolução da doença inicialmente não tratada ${ }^{16,17}$. Embora o tratamento possa proporcionar maior sobrevida, a qualidade de vida deve ser avaliada neste contexto. As recorrências ocorreram aproximadamente cinco meses após o re-tratamento, portanto, estes pacientes não se beneficiam com tratamentos de longa duração e demorada reabilitação, especialmente quando uma nova recidiva se sobrepõe às seqüelas existentes. A tentativa de cura a qualquer custo não se aplica nesta situação e a ressecabilidade do tumor não define a indicação cirúrgica, embora esta possa ser a melhor opção nas lesões iniciais.

\begin{abstract}
Objective: To evaluate the salvage treatment after local recurrence of upper aerodigestive tract squamous cell carcinoma in surgically treated patients. Methods: The charts of 943 patients who underwent primary tumor ressection and neck dissection between 1977 and 1996 were reviwed, and 107 patients with local recurrence were selected. Clinical presentation and disease control rates were evaluated. Results: Pain was the main symptom in recurrent disease. Salvage treatment was performed in $45 \%$ of patients and the extension of disease was the main cause not to perform this therapy. Salvage was performed in $54 \%$ of $r T 1 T 2$ and in $31 \%$ of rT3T4 patients $(p=0.04)$. Only four $(8 \%)$ salvaged patients were alive and disease-free more than 12 months after retreatment, all of them had a surgical salvage. The median time between initial surgery and local recurrence was eight months. New recurrences developed after a median of five months. Conclusion: Local recurrence after surgical treatment of advanced disease has a poor prognosis even in salvageable patients.
\end{abstract}

Key-words: Neoplasm recurrence, local; Head and neck neoplasms; Carcionma, squamous cells.

\section{REFERÊNCIAS}

1. Haas I, Hauser U, Ganzer U - The dilemma of follow-up in head and neck cancer patients. Eur Arch Otorhinolaryngol. 2001, 258(4):177-183.

2. Amar A, Franzi SA, Rapoport A - Evolution of patients with squamous cell carcinoma of upper aerodigestive tract. São Paulo Med J. 2003, 121(4):155-158.

3. Schwartz GJ, Mehta RH, Wenig BL, et al. - Salvage treatment for recurrent squamous cell carcinoma of the oral cavity. Head Neck. 2000, 22(1):34-41.

4. Eckardt A, Barth EL, Kokemueller H, et al. - Recurrent carcinoma of the head and neck: treatment strategies and survival analysis in a 20-year period. Oral Oncol. 2004, 40(4):427432.

5. Slaughter DP, Southwick HW, Smejkal W - Field cancerization in oral stratified squamous epithelium. Cancer. 1953, 6:963968.
6. Goodwin WJ - Salvage surgery for patients with recurrent squamous cell carcinoma of the upper aerodigestive tract: when do the ends justify the means? Laryngoscope. 2000, 110 (3 Pt 2 Suppl 93):1-18.

7. Gleich LL, Ryzenman J, Gluckman JL, et al. - Recurrent advanced (T3 or T4) head and neck squamous cell carcinoma: is salvage possible? Arch Otolaryngol Head Neck Surg. 2004, 130(1):35-38.

8. Induction chemotherapy plus radiation compared with surgery plus radiation in patients with advanced laryngeal cancer. The Department of Veterans Affairs Laryngeal Cancer Study Group. N Engl J Med. 1991, 324(24):1685-1690.

9. Ohizumi Y, Tamai Y, Imamiya S, et al. - Prognostic factors of reirradiation for recurrent head and neck cancer. Am J Clin Oncol. 2002, 25(4):408-413.

10. Spencer SA, Harris J, Wheeler RH, et al. - RTOG 96-10: reirradiation with concurrent hydroxyurea and 5-fluorouracil in patients with squamous cell cancer oh the head and neck. Int $\mathrm{J}$ Radiat Oncol Biol Phys. 2001, 51(5):1299-1304. 
11. Spencer SA, Wheeler RH, Peters GE, et al. - Concomitant chemotherapy and reirradiation as management for recurrent cancer of the head and neck. Am J Clin Oncol. 1999, 22(1):1-5.

12. O'Brien M, Schofield JB, Lorentzos A, et al. - The use of cisplatin plus 5-fluorouracil chemotherapy in an unselected group of patients with recurrent squamous cell carcinoma of the head and neck. Eur J Cancer B Oral Oncol. 1994, 30B(4):265267.

13. Choksi AJ, Hong WK, Dimery IW, et al. - Continuous cisplatin (24-hour) and 5-fluorouracil (120-hour) infusion in recurrent head and neck squamous cell carcinoma. Cancer. 1988, 61(5):909912.

14. Kohno N, Kitahara S, Kawaida M, et al. - Prognosis after salvage chemotherapy for locally unresectable recurrent squamous cell carcinoma of the head and neck. Jpn J Clin Oncol. 1999, 29(10):462-466.
15. Smit M, Balm AJ, Hilgers FJ, et al. - Pain as sign of recurrent disease in head and neck squamous cell carcinoma. Head Neck. 2001, 23(5):372-375.

16. Kowalski LP, Carvalho AL - Natural history of untreated head and neck cancer. Eur J Cancer. 2000, 36(8):1032-1037.

17. Carvalho AL, Salvajoli JV, Kowalski LP - A comparison of radiotherapy or radiochemotherapy with symptomatic treatment alone in patients with advanced head and neck carcinomas. Eur Arch Otorhinolaryngol. 2000, 257(3):164-167.

Endereço para correspondência:

Prof. Dr. Abrão Rapoport

Rua Iramaia, $n^{\circ} 136$ - Jd. Europa

CEP - 01450-020 - São Paulo - SP

E-mail:arapoport@terra.com.br 